RUNNING HEAD: Colour Perception in Grapheme-Colour Synaesthesia

\title{
The time course of synaesthetic colour perception
}

Laura Lungu $^{\mathrm{a}^{*}}$, Nicolas Rothen $^{\mathrm{b}}$, \& Devin B. Terhune $\mathrm{a}^{\mathrm{a}^{*}}$

${ }^{a}$ Department of Psychology, Goldsmiths, University of London

${ }^{\mathrm{b}}$ Faculty of Psychology, Swiss Distance University Institute, Brig, Switzerland

\section{Contributions:}

All authors developed the study concept and design. LL performed the testing and data collection. All authors analysed and interpreted the data. DBT and LL drafted the manuscript and all authors approved the final version of the manuscript for submission.

\section{Correspondence address:}

Devin B. Terhune

Department of Psychology

Goldsmiths, University of London

New Cross, London SE14 6NW, UK

E-mail: d.terhune@gold.ac.uk 


\begin{abstract}
Grapheme-colour synaesthesia is a neurodevelopmental condition wherein perception of numbers and letters consistently and involuntarily elicits the concurrent experience of a colour photism. Accumulating evidence suggests that heterogeneity in the visuospatial phenomenology of synaesthesia is attributable to the operation of top-down processes underlying photisms experienced as representations in associator synaesthetes and bottom-up processes subserving photisms experienced as spatially localized in projector synaesthetes. An untested corollary of this hypothesis is that bottom-up mechanisms will actuate earlier photism perception in projector synaesthetes. We tested this prediction in a pre-registered study in which associators and projectors completed adaptive temporal order judgment tasks for graphemes, colours, and photisms. In corroboration of the hypothesis of differential photism access across visuospatial phenomenology subtypes, projectors displayed earlier photism colour thresholds than associators whereas the two subtypes did not significantly differ in veridical colour thresholds. Synesthetes did not differ in grapheme or colour thresholds relative to non-synesthete controls, contrary to previous suggestions of superior colour processing in this condition. These results are consistent with the proposal of differential neural mechanisms underlying photism perception in subtypes of grapheme-colour synaesthesia and warrant renewed attention to heterogeneity in the mechanisms and phenomenology of this condition
\end{abstract}

Keywords: colour; synaesthesia; heterogeneity; photism; psychophysics; temporal order judgment 


\section{Introduction}

Grapheme-colour synaesthesia is a neurodevelopmental condition in which letters and numbers automatically and consistently induce the experience of a colour photism. This condition occurs in $\sim 2 \%$ of the population (Ward \& Simner, 2020) with emerging consensus for a genetic basis (Tilot et al., 2020; Ward \& Simner, 2005). Understanding this atypical condition has implications for visual awareness, learning, memory, and multisensory integration (Cohen Kadosh \& Henik, 2007; Rothen et al., 2012; Witthoft \& Winawer, 2013).

Attempts to elucidate the neural basis of synaesthesia are challenged by the perceptual heterogeneity within this condition (Dixon et al., 2004; Ward et al., 2007). In particular, synaesthetes vary in the perceived visuospatial location of their colour photisms with some experiencing photisms "in their mind's eye" (associators) and others as spatially co-localized with the inducing grapheme (projectors) (Dixon et al., 2004; Simner, 2013; Ward et al., 2007). Multiple lines of evidence have corroborated this distinction using

cognitive and perceptual tasks and suggest that projectors are impacted to a greater extent by colour photisms during selective attention tasks (Dixon et al., 2004) and can use photisms to aid visual search to a greater extent than associators (Ward et al., 2007).

One interpretation of these phenomenological differences is that they arise as a result of distinct underlying neural mechanisms (Cohen et al., 2015; van Leeuwen et al., 2011; Terhune et al., 2015a; Terhune et al., 2011). Applying dynamic causal modelling to fMRI data, van Leeuwen et al. (2011) observed that grapheme processing was characterized by a bottom-up pathway from the letter-shape area (LSA) in fusiform gyrus to V4 in projectors but a top-down pathway between these regions that was mediated by activation of superior parietal lobe (SPL) in associators (see also (Brang \& Ramachandran, 2010). One as of yet untested corollary of these differential neural mechanisms is that projectors should experience colour photisms earlier than associators. This pre-registered study investigated the time course of synaesthetic colour (photism) perception using adaptive psychophysics (https://osf.io/59gwd/). Associators and projectors completed a temporal order judgment (TOJ) task wherein they estimated whether colour photisms preceded or proceeded a reference stimulus. In order to clarify the perceptual 
specificity of any observed effects, synaesthesia subtypes and controls also completed the TOJ task with graphemes and veridical colours. Our central prediction was that projectors would display earlier thresholds than associators in the photism, but not grapheme or colour conditions. In order to evaluate the temporal locus of superior colour processing in synaesthesia (Banissy et al., 2013), we further tested the prediction that synaesthetes would display earlier thresholds in the colour, but not grapheme, conditions when compared to controls.

\section{Methods}

\section{Participants}

46 participants (24 synesthetes and 22 controls) took part in the study. Controls and synaesthetes were comparable in age (synaesthetes: range: $19-37, M=26.75, S D=5.93$; controls: range: $19-39$; $M=26.14$, $S D=5.50$ ), gender distributions (synaesthetes: 21 females [87.5\%], 3 males [12.5\%]; controls: 20 females [91\%], 2 males [9\%]), and years of post-secondary education (synaesthetes: range: $0-9 ; M=3.63, S D=2.58$; controls: range: $0-8, M=4.45, S D=2.15)$. Participants had normal or corrected-to-normal vision and no history of psychiatric or neurological disorders. Sample size was estimated via an a priori power analysis in Gpower (Erdfelder et al., 1996). A pilot study with a similar design yielded an effect size of $d=1.87$ for the contrast of photism thresholds in associators and projectors. Using this effect size and the following parameters $(1-\mathrm{B}=.90, \alpha=.05$, two-tailed, sample size ratio of $2: 1)$, the analysis required a sample of 11 associators and 5 projectors. We intended to recruit past this number (16 associators, 8 projectors [24 synaesthetes] and 24 yoked controls) to account for attrition. However, due to the COVID-19 pandemic outbreak, the study was terminated earlier, resulting in slight deviation of our control sample (15 associators, 9 projectors, 22 controls). Participants were recruited on the basis of having synaesthesia or not without any reference to their synaesthesia subtype, which was only determined at the end of the experiment to ensure that the experimenter was blind (see below). This also resulted in a slight deviation of the anticipated proportion of each subtype in the final sample size. Psychophysical data were not inspected or 
analysed until after data collection had ceased in order to prevent optional stopping. All participants provided informed consent in accordance with local ethical approval.

\section{Materials}

Grapheme-colour consistency

Consistency of grapheme-colour associations (Eagleman et al., 2007; Rothen, Seth, et al., 2013a), was assessed with the Texsyn toolbox in MATLAB (v. 2018b; MathWorks, Natick, USA) available from www.synesthete.org (Eagleman et al., 2007). The task involves the random presentation of 36 graphemes (letters from A to $\mathrm{Z}$ and numbers from 0 to 9) alongside an RGB colour palette consisting of $255 \mathrm{x} 255 \mathrm{x}$ 255 possible colours. Graphemes are presented randomly and synaesthetes are instructed to use the colour palette to select the colour that most closely approximates their photism for the respective grapheme. A "no colour" option is available for non-inducing graphemes. Controls were instructed to select the first colour that came to mind for each grapheme, without trying to remember their previous choices.

\section{Associator/projector phenomenology}

Visuospatial phenomenology of synaesthesia was measured using two self-report psychometric measures.

The Coloured Letters and Numbers (CLaN) questionnaire (Rothen, Tsakanikos, et al., 2013b) is a 16item self-report measure regarding different features of synaesthesia. Participants rated each item on a 5point Likert-scale (1: strongly disagree; 2: disagree; 3: neither agree nor disagree; 4: agree; 5: strongly agree). Scores were computed for four subscales: localisation (6 items; Cronbach's $\alpha=.94$ ), deliberate use (5 items; $\alpha=.80$ ), automaticity/attention ( 3 items; $\alpha=.82$ ), and longitudinal changes ( 3 items; $\alpha=.75$ ).

The Illustrated Synaesthetic Experiences Questionnaire (ISEQ) is a five-item questionnaire that queries synaesthetes regarding the visuospatial location of synaesthetic colour photisms (Skelton et al., 2009). Each item consists of an illustrated synaesthetic experience accompanied by a phenomenological description. Two items refer to projector experiences whereas three refer to associator experiences. Synaesthetes rate the extent to which each illustrated example accurately reflects their experience of photisms using a 7-point 
Likert scale (1: inaccurate to 7: accurate). After reverse-scoring the two projector items, the scale displayed poor internal consistency $(\alpha=.46)$.

\section{Temporal order judgment task}

In this visual task, participants judged whether a target stimulus (grapheme, colour, or photism) or a reference stimulus (cross) appeared first (see Figure 1). Against a grey background, each trial consisted of a blank inter-trial interval (600ms), a black fixation point $(200 \mathrm{~ms})$, a jittered interstimulus interval (400600ms), a target stimulus (black grapheme or coloured oval) and a concurrent reference stimulus (cross) (200ms from onset of second stimulus), an oval noise mask (200ms), a blank interval (200ms), and an untimed binary response prompt. At the prompt, participants used the mouse to select among two visually depicted options (target and reference stimuli) which had appeared first. Target and reference stimuli were presented asynchronously with the stimulus onset asynchrony (SOA) varying on a trial-by-trial basis in an adaptive manner dependent on performance, according to the adaptive PSI method (Kingdom \& Prins, 2009; see also Procedure).

The task included three conditions involving different target stimuli or perceptual judgments. In the grapheme condition, the target stimulus consisted of achromatic graphemes and participants judged whether they perceived the grapheme or cross first. The photism condition involved the same target and reference stimuli but participants judged whether they perceived the colour photism or cross first. Finally, in the colour condition, the target stimulus consisted of an oval colour patch and participants judged whether they saw the colour or the cross first. Each condition involved four different target stimuli that were presented in random order within each block. 

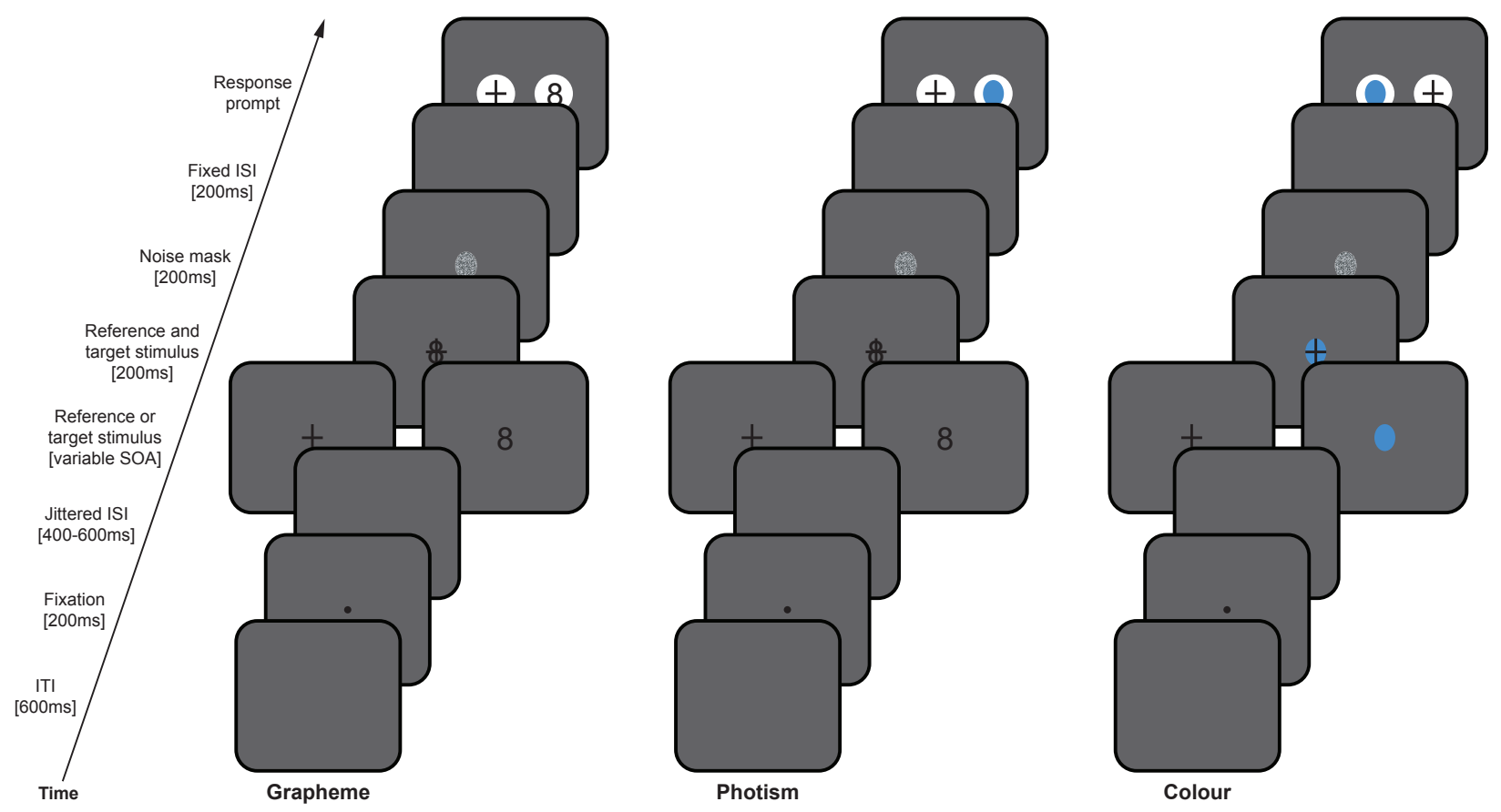

Figure 1. Temporal Order Judgement (TOJ) task. Temporal sequence of example trials in each condition. Each trial involved the presentation of two stimuli (a reference [cross] stimulus and the target [grapheme or colour patch] stimulus) with a stimulus onset asynchrony (SOA) followed by a noise mask.

Participants subsequently judged which of the two stimuli appeared first with the position of response options randomized across trials. Synaesthetes completed all three conditions whereas controls completed only the grapheme and colour conditions.

\section{Procedure}

After providing informed written consent, all participants completed the Eagleman battery (graphemecolour associations) involving 3 blocks of 36 randomly-presented graphemes. Using these data, the experimenter selected four numbers (and their associated RGB values) that displayed high consistency and had distinguishable colours for subsequent use in the TOJ task. Controls were allocated a graphemecolour set for the TOJ task based on demographic match to a specific synaesthete.

Participants subsequently completed either three conditions (synaesthetes) or two conditions (controls) of the TOJ task. Each condition consisted of one practice block of 20 trials followed by four experimental 
blocks of 50 trials, amounting to 200 trials per condition. Condition order was counterbalanced across participants and matched for synaesthete-control pairs. Prior to each block, participants were instructed to focus on the black fixation circle at the start of the trial, then pay close attention to the target and reference (cross) stimuli and then judge to the best of their ability which appeared first on the monitor. Trial-by-trial variations in the SOA between target and reference stimuli were implemented using the psi method (Kingdom \& Prins, 2009). After each trial, this method updates a Bayesian posterior distribution across a range of possible values for the threshold and slope parameters of the psychometric function. Based on this posterior distribution, the method determines for each of the possible SOAs the probabilities of observing the two different responses (target or reference) and the corresponding entropy in the posterior distribution for each response. The SOA that minimizes expected entropy in the posterior distribution is subsequently applied in the next trial.

Each condition included four adaptive sequences, corresponding to each of the four unique stimuli. These sequences were randomly interleaved to minimize participants' ability to detect response pattern structures. Stimuli and visual prompts $(1.3(\mathrm{~h}) \times 0.8(\mathrm{w}) \mathrm{cm})$ were presented at a distance of $65 \mathrm{~cm}$, subtending a visual angle of $1.1^{\circ} \times 0.7^{\circ}$. Stimulus presentation and data recording were implemented using Psychtoolbox (Brainard, 1997, Kleiner et al., 2007) and the adaptive PSI method was implemented using the Palamedes toolbox (Prins \& Kingdom, 2018), both in MATLAB. The task was presented on a 19inch iMac screen with a $60 \mathrm{~Hz}$ refresh rate.

After completing the task, synaesthetes completed the CLaN and ISEQ to determine their associator/projector status. This was done at the end of the experiment in order to ensure that the experimenter was blind to synaesthesia subtype throughout the experiment. Participants were subsequently debriefed regarding the purpose of the study.

\section{Analysis}

Grapheme-colour consistency 
Colour variation for each grapheme across the three trials was recorded using the geometric distance in RGB colour space and the data was normalized between 0 and 1. Grapheme-consistency scores were calculated using the method of Rothen et al. (2013a). RGB values were converted to CIELUV values and the Euclidean distances in CIELUV colour space for the three trials of each individual grapheme were calculated. Consistency was then computed as the mean Euclidean distance across the entire grapheme set, with lower scores reflecting greater consistency. As per previous research (Rothen et al., 2013a), scores lower than 135 were taken to denote synaesthesia.

\section{Associator/projector subtype status}

Synaesthesia subtype was determined on the basis of CLAN and ISEQ scores. Synaesthetes were classified as projectors if they scored at least a 4 out of 7 for one of the two ISEQ projector items (1 and 2), and at least a 4 out of 5 for two of the five CLAN projector questions $(2,4,7,8$, and 10). Synaesthetes that did not meet these criteria were classified as associators. These criteria were established by the authors (see OSF pre-registration).

\section{TOJ parameters}

In each TOJ condition, the median threshold and median slope were computed from the final thresholds and slopes for the four adaptive sequences involving different target stimuli. In addition, the median RT across the 200 trials in each stimulus condition was also computed.

\section{Statistical analyses}

This was a pre-registered study (https://osf.io/59gwd/) and the data are publicly available (https://osf.io/j4eau/). All analyses were performed in MATLAB and JASP (v. 0.12.2; JASP Team, Amsterdam, Netherlands). 10 univariate outliers $(M \pm 2.5 S D$ s) across the 11 dependent variables (3\%) were corrected to the corresponding outlier threshold; this included 2 grapheme thresholds (1 projector, 1 control), 2 grapheme slopes (1 projector, 1 control), 2 grapheme RTs (controls), 1 colour threshold 
(associator), 1 slope threshold (projector) and 2 colour RT (controls). There were no outliers among the three dependent measures in the photism condition or in the threshold difference measures.

The primary analyses consisted of hypothesis-focused independent Welch t-tests comparing associators and projectors on the central dependent variable of interest (photism thresholds), control $t$-tests comparing these two subtypes on grapheme and colour thresholds and condition difference (photism-grapheme and photism-colour) thresholds, and finally a hypothesis-driven $t$-test comparing synaesthetes and controls on colour thresholds. Effect sizes consisted of Hedges's gs and Bootstrap 95\% CIs (10,000 samples, biascorrected and accelerated method; Efron, 1987). In order to test two central null hypotheses of interest, namely that associators and projectors would not differ in grapheme and colour thresholds, we computed Bayes factors for these differences using the corresponding difference between subtypes in photism thresholds as a prior. Bayes factors were computed in MATLAB using half-normal distributions $\left(B F_{0, S D}\right)$ where $S D$ corresponds to the prior (Dienes, 2014). BFs provide an estimate of the relative likelihood of one hypothesis relative to another (Dienes, 2014); following convention (Dienes, 2011; Jeffreys, 1961), we interpret BFs less than 0.33 or greater than 3 as reflecting moderate evidence for the null and alternative hypotheses, respectively, and in-between values as reflecting insensitive evidence.

For completeness, analyses of thresholds were repeated using a series of mixed-model ANOVAs with different between-groups independent variables (Subtype: associator vs. projector; Group: associator vs. projector vs. control), and within-groups independent variables (grapheme vs. colour vs. photism [synaesthetes only]). Greenhouse-Geisser corrections were applied when data violated the assumption of sphericity. Secondary and exploratory analyses are described in the Supplementary Materials.

\section{Results}

\section{Grapheme-colour consistency}

Grapheme-colour consistency is widely used as a method to corroborate synaesthesia (Eagleman et al., 2007; Rothen et al., 2013a). All synaesthetes displayed averaged distances in CIELUV colour space below the threshold for synaesthesia (Rothen et al., 2013a), $M=64, S D=27$, whereas none of the controls did, 
$M=306, S D=52$. Synaesthetes' scores were significantly lower than those of controls, $t(30.93)=19.35$, $p<.001, g=5.76[4.72,7.85]$, whereas associators, $M=63, S D=26$, did not significantly differ from projectors, $M=66, S D=31, t(14.56)=0.24, p=.81, g=0.10[-0.75,1.02]$. These results corroborate the presence of synaesthesia in these synaesthetes.

\section{Pre-registered analyses}

Pre-registered analyses consisted of primary analyses directly pertaining to the effects of interest including photism threshold differences across synaesthesia subtypes and secondary analyses of slopes and RTs.

\section{Primary analyses}

\section{Photism thresholds}

As predicted, projectors, $M=84 \mathrm{~ms}, S D=106$, displayed significantly earlier photism thresholds than associators, $M=217 \mathrm{~ms}, S D=163, t(21.74)=2.41, p=.025$, corresponding to a large effect, $g=0.88[0.25$, 1.58] (see Figure 2). The magnitude of this effect was only slightly attenuated or similar when using difference threshold scores, accounting for grapheme thresholds (photism-grapheme), $t(21.63)=1.76$, $p=.093, g=0.65[-0.06,1.32]$, and colour thresholds (photism-colour), $t(21.90)=2.57, p=.018, g=0.93[0.31$, 1.61]. 


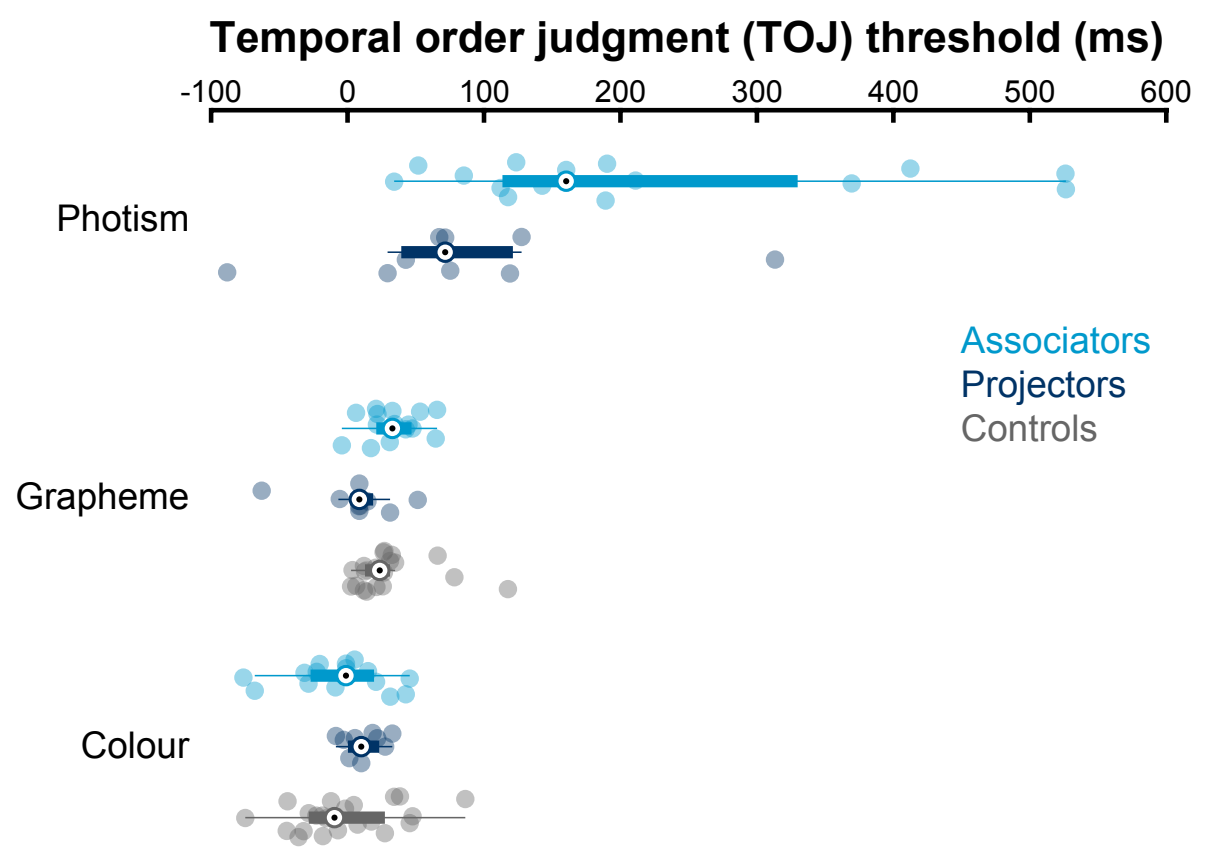

Figure 2. Box plots and individual values for TOJ thresholds as a function of Condition and Group. Controls did not complete the photism condition. Positive thresholds indicate that the target stimulus (photism, grapheme, or colour) was perceived later than the reference stimulus (cross) whereas negative thresholds indicate that the target stimulus was perceived earlier than the reference stimulus.

\section{Grapheme thresholds}

We expected that the three groups would not differ in grapheme thresholds. As predicted, controls, $M=29$, $S D=27$, had comparable thresholds relative to synaesthetes, $M=25, S D=32$, and did not significantly differ, $t(43.77)=0.42, p=.67, g=0.12[-0.49, .64]$. Unexpectedly, projectors displayed earlier grapheme thresholds, $M=7, S D=32$, than associators, $M=36, S D=27, t(14.68)=2.32, p=.035, g=0.99[0.35,1.79]$. Using the associator-projector difference in photism thresholds as a prior, $M=133$, we found evidence that the subtype difference in grapheme thresholds, $M=29, S E=12$, was consistent with the hypothesis, that the two subtypes differ, $B F_{0,133}=3.23$. However, it is notable that projectors did not significantly differ from controls, $t(12.92)=1.83, p=.091, g=0.76[0.07,1.47]$; similarly associators and controls did not significantly differ, $t(30.35)=0.82, p=.42, g=0.27[-0.32,1.09]$. 


\section{Colour thresholds}

We did not expect associators and projectors to differ in colour thresholds although we suspected that synaesthetes would exhibit earlier colour thresholds than controls. In contrast with the photism threshold results, projectors tended to display significantly later colour thresholds, $M=12, S D=14$, than associators, $M=-1, S D=36$, although this did not achieve significance, $t(19.96)=1.75, p=.096, g=0.59[0.05,1.33]$. As above, we used the associator-projector difference in photism thresholds as a prior, $M=133$, and found that there was evidence that the subtype difference in colour thresholds, $M=-13, S E=9$, was consistent with the null hypothesis that the two subtypes do not differ, $B F_{0,133}=0.027$. In addition, the expected colour advantage in synaesthetes did not hold, as controls, $M=-2, \mathrm{SD}=38$, exhibited similar colour thresholds relative to synaesthetes, $M=0, S D=30, t(40.35)=0.80, p=.91, g=0.08[-0.48,0.73]$.

\section{Factorial analyses}

For completeness, we repeated these analyses using three mixed-model ANOVAs with Condition as a repeated-measures variable and subtype (associator vs. projector) and group (control vs. synaesthete and control vs. associator vs. projector) as between-groups factors.

The 3 (Condition) x 2 (Synaesthesia subtype) mixed-model ANOVA on thresholds yielded a significant effect of Condition, $F(1.09,23.88)=17.66, p<.001, \eta_{p}^{2}=.45$, a significant Subtype effect, $F(1,22)=6.29, p=.020, \eta_{p}^{2}=.22$, which were moderated by a marginal Condition $\mathrm{x}$ Subtype interaction, $F(1.09,23.88)=4.05, p=.053, \eta_{p}^{2}=.16$. Post hoc Tukey tests revealed that this interaction was driven by significantly earlier thresholds among projectors than associators in the photism condition, $M=133, S E=37$, $t=3.62, p=.007$, but not in the grapheme, $M=29, S E=37, t=0.80, p=.97$, or the colour condition, $M=-18$, $S E=37, t=0.49, p=1.00$ (for all contrasts, see Supplementary Materials, Table 2).

The 2 (Condition) x 3 (Group) mixed-model ANOVA on thresholds yielded a significant Condition effect, $F(1,43)=13.31, p<.001, \eta_{p}^{2}=.24$, a non-significant Group effect with a miniscule effect size, $F(2,43)=0.16, p=.85, \eta_{p}^{2}<.01$, which were moderated by a Condition x Group interaction, $F(2,43)=4.15$, 
$p=.022, \eta^{2}=.16$. Post hoc Tukey tests revealed that this interaction seemed to be driven by significantly later thresholds in the grapheme than the colour conditions in both controls, $M=31, S E=8, t=3.70, p=.005$, and associators, $M=42, S E=10, t=4.14, p=.002$. By contrast, projectors displayed relatively similar thresholds in the two conditions, $M=5, S E=13, t=0.38, p=0.99$ (for all contrasts and analyses collapsing across synaesthesia subtypes, see Supplementary Materials, Table 3).

\section{Secondary and exploratory analyses}

Secondary analyses were performed on psychometric function slopes and RTs in the different conditions in controls and synaesthetes. These analyses did not reveal any significant differences between groups or synaesthesia subtypes. They further suggested that synaesthetes displayed lower (i.e., flatter) slopes and slower RTs in the photism condition relative to the grapheme and colour conditions (see Supplementary Results).

Exploratory correlations assessed associations between TOJ thresholds in the three conditions and psychometric measures pertaining to synaesthesia phenomenology. Across synaesthetes, photism thresholds were weakly, albeit non-significantly correlated with self-reported synaesthesia phenomenology (see Supplementary Results).

\section{Discussion}

By coupling a temporal-order judgment (TOJ) task with adaptive psychophysics, our results corroborate the hypothesis that projector synaesthetes experience colour photisms earlier than associator synaesthetes. Further analyses suggest that this effect was independent of TOJ thresholds for graphemes and colours and thus this difference between subtypes seems to be specific to photism perception and not reflective of a generic colour processing benefit or bias in projectors. These results corroborate the view that synaesthesia subtypes experience colour photisms through distinct neurophysiological mechanisms (van Leeuwen et al., 2011) and have implications for the neural basis of synaesthesia (Ward, 2013). 
Earlier photism perception in projectors relative to associators complements the previous observation that projector synaesthesia seems to occur through a bottom-up (direct) neural pathway - from LSA to V4 - whereas associator synaesthesia involves a top-down (indirect) pathway involving intermediary processing by SPL before V4 activation (van Leeuwen et al., 2011). Our results suggest the latter route produces an average delay of $\sim 133 \mathrm{~ms}$ although this difference was variable in our sample. The relatively early time course of photism perception in projectors is also conceptually congruent with a magnetoencephalography study of projectors showing V4 activation $\sim 114 \mathrm{~ms}$ after grapheme presentation (Brang et al., 2010). The specific time course of photism awareness in these different groups remains undetermined but could be addressed more rigorously using other methods such as continuous flash suppression (Tsuchiya \& Koch, 2005). These various effects may be subserved by enhanced parietal cortex power in the alpha band frequency and/or enhanced primary visual cortex excitability in projectors relative to associators (Cohen et al., 2015; Terhune et al., 2015a; Terhune et al., 2015b). Insofar as the two subtypes did not differ in colour thresholds - with Bayesian evidence in favor of the null hypothesis of no difference - it appears that these differential pathways between associators and projectors are specific to colour photisms and not reflective of broader differences in colour processing between these subtypes.

The observation of earlier photism perception in projectors can help to understand various unique characteristics in this subgroup. Previous research studying the "pop-out effect" indicated that projector synaesthetes experience colours more reliably relative to associators and that projectors are more likely to be aware of their photisms during brief presentations (Ward et al., 2010; Palmeri et al., 2002; Smilek et al., 2003). Accordingly, synaesthetes displaying earlier photism thresholds in the present study might be expected to display earlier photism awareness in such paradigms. Similarly, the previous observation that projectors exhibit greater Stroop interference from photisms during veridical colour naming (Dixon et al., 2004) is plausibly attributable to earlier photism awareness in this subgroup.

An unexpected result was that projectors displayed slightly earlier $(\sim 29 \mathrm{~ms})$ grapheme thresholds than associators. Although this effect was significant when performing direct comparisons, it did not survive a post hoc Tukey correction. Additionally, neither associators' nor projectors' grapheme thresholds 
significantly differed from those of controls indicating that projectors do not seem to display atypical grapheme processing. Taken together, these results suggest that the difference observed between associators and projectors is not reflective of a robust dissimilarity. This is in line with its notably smaller magnitude relative to the differential photism thresholds effect in synaesthesia subtypes ( $\sim 133 \mathrm{~ms})$, although Bayesian statistics showed that the former was consistent with the latter. Moreover, among synaesthetes, grapheme and photism thresholds did not significantly correlate $\left(r_{\mathrm{s}}=.09\right.$; see Supplementary Materials). In addition, the photism threshold difference between subtypes remained strong in magnitude $(g=0.65)$ after correcting for grapheme thresholds. Taken together, these results suggest that earlier grapheme and photism thresholds among projectors reflect independent effects. Further research is required to replicate this unpredicted difference between subtypes.

Controls and synaesthetes did not reliably differ in grapheme or stimulus colour processing with uniformly small effect sizes. This suggests that synaesthetes do not exhibit atypical conscious access of graphemes relative to controls. The lack of a group difference in the stimulus colour condition is potentially surprising given the multitude of studies highlighting superior colour processing in synaesthetes (e.g., Banissy et al., 2013). This apparent discrepancy is potentially because we assessed temporal order characteristics of colour processing. Although we could not confirm differences in processing speed for veridical colours between synaesthesia subtypes or between synaesthetes and controls, this does not exclude the possibility that synaesthesia confers an advantage in colour discrimination.

In summary, our results strongly suggest that projector synaesthetes experience colour photisms earlier than associator synaesthetes, thereby corroborating the hypothesis that projectors process synaesthetic colours through a direct, bottom-up pathway whereas associators process synaesthetic colours more indirectly through a top-down pathway (Brang \& Ramachandran, 2010; van Leeuwen et al., 2011). These results help to explain the impact of differential visuospatial phenomenology of colour photisms on a range of cognitive-perceptual tasks in synaesthetes (Ward et al., 2010) and reinforces the importance of elucidating the characteristics and mechanisms underlying heterogeneity in this condition. 


\section{Acknowledgments}

We thank Jamie Ward with assistance in recruiting synaesthetes.

\section{References}

Banissy, M. J., Tester, V., Muggleton, N. G., Janik, A. B., Davenport, A., Franklin, A., Walsh, V., \& Ward, J. (2013). Synesthesia for color is linked to improved color perception but reduced motion perception. Psychological Science, 24(12), 2390-2397.

Brainard, D. H. (1997). The psychophysics toolbox. Spatial Vision, 10(4), 433-436.

Brang, D., \& Ramachandran, V. S. (2010). Visual field heterogeneity, laterality, and eidetic imagery in synesthesia. Neurocase, 16(2), 169-174.

Cohen Kadosh, R., \& Henik, A. (2007). Can synaesthesia research inform cognitive science? Trends in Cognitive Sciences, 11(4), 177-184.

Cohen, M. X., Weidacker, K., Tankink, J., Scholte, H. S., \& Rouw, R. (2015). Grapheme-color synesthesia subtypes: Stable individual differences reflected in posterior alpha-band oscillations. Cognitive Neuroscience, 6(2-3), 56-67.

Dienes, Z. (2011). Bayesian versus orthodox statistics: Which side are you on? Perspectives on Psychological Science, 6(3), 274-290. doi: 10.1177/1745691611406920

Dienes, Z. (2014). Using Bayes to get the most out of non-significant results. Frontiers in Psychology, 5, 781. doi: 10.3389/fpsyg.2014.00781

Dixon, M. J., Smilek, D., \& Merikle, P. M. (2004). Not all synaesthetes are created equal: projector versus associator synaesthetes. Cognitive, Affective \& Behavioral Neuroscience, 4(3), 335-343.

Eagleman, D. M., Kagan, A. D., Nelson, S. S., Sagaram, D., \& Sarma, A. K. (2007). A standardized test battery for the study of synesthesia. Journal of Neuroscience Methods, 159(1), 139-145.

Efron, B. (1987). Better bootstrap confidence intervals. Journal of the American Statistical Association 82(397), 171-185. https://doi.org/10.2307/2289153 
Erdfelder, E., Faul, F., \& Buchner, A. (1996). GPOWER: A general power analysis program. Behavior Research Methods, Instruments, \& Computers, 28(1), 1-11. https://doi.org/10.3758/bf03203630 Jeffreys, H. (1961). The theory of probability (3rd ed.). Oxford University Press.

Kingdom, F. A. A., \& Prins, N. (2009). Psychophysics: A Practical Introduction. Academic Press.

Kleiner M, Brainard D, Pelli D. (2007). What's new in Psychtoolbox-3? Perception, 36, 1-16.

Palmeri, T. J., Blake, R., Marois, R., Flanery, M. A., \& Whetsell, W., Jr. (2002). The perceptual reality of synesthetic colors. Proceedings of the National Academy of Sciences of the United States of America, 99(6), 4127-4131.

Prins, N., \& Kingdom, F. A. A. (2018). Applying the model-comparison approach to test specific research hypotheses in psychophysical research using the Palamedes Toolbox. Frontiers in Psychology, 9:1250. doi:10.3389/fpsyg.2018.01250

Rothen, N., Meier, B., \& Ward, J. (2012). Enhanced memory ability: Insights from synaesthesia. Neuroscience and Biobehavioral Reviews, 36(8), 1952-1963.

Rothen, N., Seth, A. K., Witzel, C., \& Ward, J. (2013a). Diagnosing synaesthesia with online colour pickers: maximising sensitivity and specificity. Journal of Neuroscience Methods, 215(1), 156-160.

Rothen, N., Tsakanikos, E., Meier, B., \& Ward, J. (2013b). Coloured Letters and Numbers (CLaN): a reliable factor-analysis based synaesthesia questionnaire. Consciousness and Cognition, 22(3), $1047-1060$.

Simner, J. (2013). Why are there different types of synesthete? Frontiers in Psychology, 4, 558.

Skelton, R., Ludwig, C., \& Mohr, C. (2009). A novel, illustrated questionnaire to distinguish projector and associator synaesthetes. Cortex, 45(6), 721-729.

Smilek, D., Dixon, M. J., \& Merikle, P. M. (2003). Synaesthetic photisms guide attention. Brain and Cognition, 53(2), 364-367.

Terhune, D. B., Murray, E., Near, J., Stagg, C. J., Cowey, A., \& Cohen Kadosh, R. (2015a). Phosphene perception relates to visual cortex glutamate levels and covaries with atypical visuospatial awareness. Cerebral Cortex, 25(11), 4341-4350. 
Terhune, D. B., Song, S. M., \& Cohen Kadosh, R. (2015b). Transcranial alternating current stimulation reveals atypical $40 \mathrm{~Hz}$ phosphene thresholds in synaesthesia. Cortex, 63, 267-270.

Terhune, D. B., Tai, S., Cowey, A., Popescu, T., \& Cohen Kadosh, R. (2011). Enhanced cortical excitability in grapheme-color synesthesia and its modulation. Current Biology, 21(23), 2006-2009.

Tilot, A. K., Vino, A., Kucera, K. S., Carmichael, D. A., Van den Heuvel, L., Den Hoed, J., ... \& Van Leeuwen, T. M. (2019). Investigating genetic links between grapheme-colour synaesthesia and neuropsychiatric traits. Philosophical Transactions of the Royal Society of London B: Biological Sciences, 374(1787), 20190026.

Tsuchiya, N., \& Koch, C. (2005). Continuous flash suppression reduces negative afterimages. Nature Neuroscience, 8(8), 1096-1101.

van Leeuwen, T. M., den Ouden, H. E. M., \& Hagoort, P. (2011). Effective connectivity determines the nature of subjective experience in grapheme-color synesthesia. Journal of Neuroscience, 31(27), 9879-9884.

Ward, J., Jonas, C., Dienes, Z., \& Seth, A. (2010). Grapheme-colour synaesthesia improves detection of embedded shapes, but without pre-attentive "pop-out" of synaesthetic colour. Philosophical Transactions of the Royal Society of London B: Biological Sciences, 277(1684), 1021-1026.

Ward, J., Li, R., Salih, S., \& Sagiv, N. (2007). Varieties of grapheme-colour synaesthesia: a new theory of phenomenological and behavioural differences. Consciousness and Cognition, 16(4), 913-931.

Ward, J., \& Simner, J. (2005). Is synaesthesia an X-linked dominant trait with lethality in males? Perception, 34(5), 611-623.

Ward, J., \& Simner, J. (2020). Synesthesia. In K. Sathian \& V. S. Ramachandran (Eds.), Multisensory perception: From laboratory to clinic (pp. 283-300). Academic Press. https://doi.org/10.1016/b978$0-12-812492-5.00013-9$

Witthoft, N., \& Winawer, J. (2013). Learning, memory, and synesthesia. Psychological Science, 24(3), 258-265. 


\section{Supplementary Information}

\section{The time course of synaesthetic colour perception}

Laura Lungu ${ }^{\mathrm{a}^{*}}$, Nicolas Rothen ${ }^{\mathrm{b}}, \&$ Devin B. Terhune $\mathrm{a}^{\mathrm{a}^{*}}$

${ }^{a}$ Department of Psychology, Goldsmiths, University of London

${ }^{\mathrm{b}}$ Faculty of Psychology, Swiss Distance University Institute, Brig, Switzerland

\section{Correspondence address:}

Devin B. Terhune, d.terhune@gold.ac.uk

Condition threshold effects as a function of synaesthesia subtype (associators vs. projectors)

Supplementary Table 1 reports post hoc Tukey tests comparing grapheme, photism, and colour thresholds across Conditions and Subtypes (see also main text for descriptions of the corresponding ANOVA).

\section{Supplementary Table 1.}

Post hoc comparisons of condition thresholds in associators and projectors

\begin{tabular}{|c|c|c|c|c|c|c|}
\hline & & $M_{\text {difference }}$ & $S E$ & $t$ & $d$ & $p$ \\
\hline \multirow[t]{5}{*}{ Colour, Associator } & Colour, Projector & -0.018 & 0.037 & -0.492 & -0.101 & .996 \\
\hline & Grapheme, Associator & -0.042 & 0.033 & -1.277 & -0.261 & .795 \\
\hline & Grapheme, Projector & -0.013 & 0.037 & -0.357 & -0.073 & .999 \\
\hline & Photism, Associator & -0.223 & 0.033 & -6.728 & -1.373 & $<.001$ \\
\hline & Photism, Projector & -0.090 & 0.037 & -2.469 & -0.504 & .149 \\
\hline \multirow[t]{4}{*}{ Colour, Projector } & Grapheme, Associator & -0.024 & 0.037 & -0.664 & -0.135 & .985 \\
\hline & Grapheme, Projector & -0.005 & 0.043 & -0.116 & -0.024 & 1.000 \\
\hline & Photism, Associator & -0.205 & 0.037 & -5.596 & -1.142 & $<.001$ \\
\hline & Photism, Projector & -0.072 & 0.043 & -1.692 & -0.345 & .544 \\
\hline \multirow[t]{3}{*}{ Grapheme, Associator } & Grapheme, Projector & 0.029 & 0.037 & 0.799 & 0.163 & .967 \\
\hline & Photism, Associator & -0.181 & 0.033 & -5.450 & -1.113 & $<.001$ \\
\hline & Photism, Projector & -0.048 & 0.037 & -1.313 & -0.268 & .777 \\
\hline \multirow[t]{2}{*}{ Grapheme, Projector } & Photism, Associator & -0.210 & 0.037 & -5.731 & -1.170 & $<.001$ \\
\hline & Photism, Projector & -0.077 & 0.043 & -1.807 & -0.369 & .567 \\
\hline Photism, Associator & Photism, Projector & 0.133 & 0.037 & 3.619 & 0.739 & .007 \\
\hline
\end{tabular}

Notes. $p$-values reflect Tukey correction for multiple comparisons. 
Condition threshold effects as a function of Group (controls vs. associators vs. projectors)

Supplementary Table 2 reports post hoc Tukey tests comparing grapheme and colour thresholds across Conditions and Groups (see also main text for descriptions of the corresponding ANOVA).

Supplementary Table 2.

Post hoc comparisons of condition thresholds in controls, associators, projectors.

\begin{tabular}{|c|c|c|c|c|c|c|}
\hline & & $M_{\text {difference }}$ & $S E$ & $t$ & $d$ & $p$ \\
\hline \multirow[t]{5}{*}{$\overline{\text { Control-Colour }}$} & Associator-Colour & 0.004 & 0.010 & 0.398 & 0.059 & .999 \\
\hline & Projector-Colour & -0.014 & 0.012 & -1.139 & -0.168 & .863 \\
\hline & Control-Grapheme & -0.031 & 0.008 & -3.657 & -0.539 & .008 \\
\hline & Associator-Grapheme & -0.038 & 0.010 & -3.698 & -0.545 & .005 \\
\hline & Projector-Grapheme & -0.009 & 0.012 & -0.734 & -0.108 & .977 \\
\hline \multirow[t]{4}{*}{ Associator-Colour } & Projector-Colour & -0.018 & 0.013 & -1.385 & -0.204 & .736 \\
\hline & Control-Grapheme & -0.035 & 0.010 & -3.386 & -0.499 & .013 \\
\hline & Associator-Grapheme & -0.042 & 0.010 & -4.139 & -0.610 & .002 \\
\hline & Projector-Grapheme & -0.013 & 0.013 & -1.005 & -0.148 & .915 \\
\hline \multirow[t]{3}{*}{ Projector-Colour } & Control-Grapheme & -0.017 & 0.012 & -1.389 & -0.205 & .733 \\
\hline & Associator-Grapheme & -0.024 & 0.013 & -1.867 & -0.275 & .429 \\
\hline & Projector-Grapheme & 0.005 & 0.013 & 0.375 & 0.055 & .999 \\
\hline \multirow[t]{2}{*}{ Control-Grapheme } & Associator-Grapheme & -0.007 & 0.010 & -0.710 & -0.105 & .980 \\
\hline & Projector-Grapheme & 0.022 & 0.012 & 1.795 & 0.265 & .475 \\
\hline Associator-Grapheme & Projector-Grapheme & 0.029 & 0.013 & 2.248 & 0.331 & .228 \\
\hline
\end{tabular}

Notes. $p$-values reflect Tukey correction for multiple comparisons.

Condition threshold effects as a function of Group (controls vs. synaesthetes)

The 2 (Condition) x 2 (Group) mixed-model ANOVA on thresholds yielded a main effect of Condition, $F(1,44)=19.42, p<.001, \eta^{2} p^{2} .31$, which reflected faster colour than grapheme thresholds. By contrast, the main effect of Group, $F(1,44)<0.01, p=.94, \eta^{2}{ }_{p}<.01$, and the Condition x Group interaction, $F(1,44)=0.25, p=.62, \eta^{2}=.01$, were both non-significant with notably small effect sizes. This suggests that participants displayed earlier colour thresholds than grapheme thresholds but that this difference was not significantly moderated by group. 


\section{Exploratory analyses}

We performed two sets of exploratory analyses: (1) repeating the analyses of condition thresholds on condition slopes and RTs and (2) assessing correlations between condition thresholds and psychometric measures.

\section{Analyses of condition slopes}

Associators, $M=0.67, S D=0.35$, and projectors, $M=0.79, S D=0.28$, did not significantly differ in slopes in the photism condition, $t(20.23)=0.89, p=.38, g=0.34[-0.42,1.22]$. The same held for the grapheme condition: associators, $M=1.12, S D=0.27$, projectors, $M=1.08, S D=0.36, t(13.58)=0.32, p=.76, g=0.14$ $[-0.86,0.99]$, and the colour condition: associators, $M=0.94, S D=0.31$, projectors, $M=1.08, S D=0.36$, $t(14.99)=1.04, p=.32, g=0.44[-0.39,1.43]$. Similarly, controls and synaesthetes did not significantly different in either grapheme slopes: controls, $M=1.10, S D=0.28$, synaesthetes, $M=1.10, S D=0.30$, $t(43.98)=0.07, p=.94, g=0.02[-0.58,0.58]$, or colour slopes: controls, $M=0.94, S D=0.30$, synaesthetes, $M=0.99, S D=0.33, t(43.99)=0.61, p=.55, g=0.18[-0.42,0.74]$.

The 3 (Condition) x 2 (Synaesthesia subtype) mixed-model ANOVA on slopes yielded a significant effect of Condition, $F(1.61,35.44)=9.96, p<.001, \eta^{2}{ }^{2}=.31$, but a non-significant Subtype effect, $F(1,22)=0.67, p=.42, \eta_{p}=.03$, and a non-significant Condition x Subtype interaction, $F(1.61,35.44)=0.70, p=.47, \eta^{2}=.03$. Post hoc Bonferroni-corrected contrasts indicated that the Condition effect reflected flatter slopes in the photism condition than both the colour condition, $M_{\text {difference }}=0.28, S E=0.10, t=2.91, p=.02, d=0.59$, and the grapheme condition, $M=0.39, S E=0.09$, $t=4.42, p<.001, d=0.90$, which did not significantly differ, $M=-0.11, S E=0.06, t=1.80, p=.26, d=0.37$.

The 2 (Condition) x 3 (Group) ANOVA on slopes yielded similar results: a significant effect of Condition, $F(1,43)=4.93, p=.032, \eta^{2}{ }^{2}=.10$, reflecting steeper slopes in the grapheme than colour condition, but a non-significant Group effect, $F(2,43)=0.20, p=.82, \eta_{p}<.01$, and Condition $\mathrm{x}$ Group interaction, $F(2,43)=1.12, p=.34, \eta^{2}=.05$. When this analysis was repeated collapsing across subtype, it yielded similar results: Condition: $F(1,44)=8.10, p=.007, \eta^{2}{ }_{p}=.16$; Group: $F(1,44)=0.17, p=.68$, $\eta_{p}^{2}<.01$; Condition x Group: $F(1,44)=0.27, p=.61, \eta^{2}=.01$. 
Analyses of condition RTs

Associators, $M=1000 \mathrm{~ms}, S D=493$, and projectors, $M=966 \mathrm{~ms}, S D=484$, did not significantly differ in RTs in the photism condition, $t(17.22)=0.16, p=.87, g=0.06[-0.79,0.85]$. The same held for the grapheme condition: associators: $M=733 \mathrm{~ms}, S D=207$, and projectors: $M=810 \mathrm{~ms}, S D=190$, $t(18.20)=0.93, p=.37, g=0.37$ [-0.37, 1.37], and the colour condition: associators: $M=730 \mathrm{~ms}, S D=191$, and projectors: $M=800 \mathrm{~ms}, S D=206, t(15.97)=0.83, p=.42, g=0.34[-0.46,1.11]$. Similarly, controls and synaesthetes did not significantly different in grapheme RTs: controls, $M=758 \mathrm{~ms}, S D=252$, synaesthetes, $M=762 \mathrm{~ms}, S D=200, t(40.06)=0.05, p=.96, g=0.01[-0.52,0.69]$, or colour RTs: controls, $M=741 \mathrm{~ms}, S D=216$, synaesthetes, $M=757, S D=195, t(42.49)=0.26, p=.80, g=0.08 \quad[-0.50,0.67]$. Collectively, these data suggest that the groups did not significantly differ in RTs in the different conditions.

The 3 (Condition) x 2 (Synaesthesia subtype) mixed-model ANOVA on RTs yielded a significant effect of Condition, $F(1.13,24.74)=5.34, p=.026, \eta^{2}{ }^{2}=.20$, but a non-significant Subtype effect, $F(1,22)=0.13, p=.73, \eta^{2}=.01$, and a non-significant Condition x Subtype interaction, $F(1.13,24.74)=0.33, p=.60, \eta^{2}=.02$. Post hoc Bonferroni-corrected contrasts indicated that the Condition effect reflected slower RTs in the photism condition than both the colour condition, $M_{\text {difference }}=-230 \mathrm{~ms}, S E=88, t=2.61, p=.047, d=0.53$, and the grapheme condition, $M=-225, S E=86$, $t=2.63, p=.044, d=0.54$, which did not significantly differ, $M=5, S E=25, t=0.20, p=1.00, d=0.04$.

The 2 (Condition) x 3 (Group) ANOVA on RTs yielded all non-significant results with miniscule effect sizes: Condition, $F(1,43)=0.24, p=.63, \eta^{2}=.01$; Group: $F(2,43)=0.36, p=.70, \eta_{p}=.02$; Condition x Group: $F(2,43)=0.06, p=.94, \eta^{2}{ }^{2}<.01$. When this analysis was repeated collapsing across subtype, it yielded similar results: Condition: $F(1,44)=0.37, p=.55, \eta^{2}{ }_{p}=.01$; Group: $F(1,44)=0.03, p=.88, \eta^{2}{ }_{p}<.01$; Condition x Group: $F(1,44)=0.11, p=.74, \eta^{2}{ }_{p}<.01$.

In the first instance, these results suggest that associators and projectors did not exhibit significantly different slopes or RTs in the various TOJ conditions, implying relatively similar levels of precision and general performance. In particular, projectors were non-significantly faster in the photism condition suggesting that differential photism thresholds are unlikely to be attributable to a speed-accuracy tradeoff. However, the two groups similarly displayed flatter slopes and slower RTs in 
the photism condition relative to the other conditions, suggesting that the increased difficulty of making judgments about mental representations than stimulus-driven percepts leads to poorer precision and slower responses. Taken together, this suggests that photism thresholds differences between subtypes are unlikely to be driven by differential precision or response latencies.

In contrast with our expectations, controls and synaesthetes did not reliably differ in the TOJ conditions in either precision or response latencies. In particular, they displayed comparable slopes and RTs in both the grapheme and colour conditions with reliably weak effect sizes. Notably, both groups displayed steeper slopes in the grapheme than the colour condition, without corresponding differences in RTs, suggesting perhaps superior precision for grapheme awareness judgments.

\section{Correlations between psychophysical thresholds and psychometric measures}

Supplementary Table 3 presents Spearman skipped correlations between psychophysical thresholds in the three TOJ conditions (grapheme, colour, photism) and scores on different synaesthesia psychometric measures in synaesthetes. We expected that photism thresholds would be correlated with the localisation factor of the CLaN (CLaN:L) and the total ISEQ score.

In corroboration of the CLaN localisation factor, this measure significantly correlated with the ISEQ, $r_{\mathrm{s}}=.46$. As predicted, photism thresholds were weakly negatively correlated with CLaN:L, $r_{\mathrm{s}}=-$ .37 , and ISEQ scores, $r_{\mathrm{s}}=-.33$, although neither of these effects achieved significance. Similarly, photism thresholds did significantly correlate with any other CLaN factor, $r_{\mathrm{s}}<|.28|$, and the ISEQ did not significantly correlate with any other condition thresholds or CLaN factor scores, $r_{\mathrm{s}}<|.41|$. An unexpected result was that grapheme thresholds significantly negatively correlated with CLaN:L factor scores, $r_{\mathrm{s}}=-.50$, which is potentially consistent with differential grapheme thresholds in associators and projectors (see main text). CLaN factor scores did not significantly inter-correlate, or correlate with any other condition thresholds, $r_{\mathrm{s}}<|.32|$. Similarly, grapheme-colour consistency scores did not significantly correlate with any of the other measures, $r_{\mathrm{s}}<|.32|$. Owing to the small sample size, and lack of correction for multiple comparisons, these correlations should be treated with caution but suggest potential links between grapheme and photism thresholds and psychometric measures of photism localisation in synaesthesia that are worth pursuing further in future research. 


\section{Supplementary Table 3.}

Spearman (skipped) correlations between condition thresholds and psychometric measures in grapheme-colour synaesthetes $(N=24)$

\begin{tabular}{|c|c|c|c|c|c|c|c|c|c|}
\hline & $\begin{array}{c}\text { Photism } \\
\text { threshold } \\
\text { (PT) }\end{array}$ & $\begin{array}{c}\text { Grapheme } \\
\text { Threshold } \\
\text { (GT) }\end{array}$ & $\begin{array}{c}\text { Colour } \\
\text { Threshold } \\
\text { (CT) }\end{array}$ & CLaN:L & CLaN:DU & CLaN:A & CLaN:LC & ISEQ & Consistency \\
\hline$\overline{\mathrm{PT}}$ & & $\begin{array}{c}.09[-.36, \\
.55]\end{array}$ & $\begin{array}{c}-.02[-.42, \\
.40]\end{array}$ & $\begin{array}{c}-.37[-.71 \\
.06]\end{array}$ & $\begin{array}{c}.09[-.40, \\
.54]\end{array}$ & $\begin{array}{l}-.27[-.64, \\
.19]\end{array}$ & $\begin{array}{l}-.12[-.57, \\
.27]\end{array}$ & $\begin{array}{l}-.33[-.64, \\
.07]\end{array}$ & $.14[-.30, .56]$ \\
\hline GT & & & $\begin{array}{c}.03[-.46 \\
.44]\end{array}$ & $\begin{array}{c}-.50[-.73,- \\
.14]^{*}\end{array}$ & $\begin{array}{c}.02[-.39 \\
.48]\end{array}$ & $\begin{array}{l}.28[-.16, \\
.67]\end{array}$ & $\begin{array}{l}-.23[-.61, \\
.18]\end{array}$ & $\begin{array}{l}-.41[-.72, \\
.02]\end{array}$ & $\begin{array}{l}-.29[-.65, \\
.27]\end{array}$ \\
\hline CT & & & & $.35[-.08, .70]$ & $\begin{array}{c}.32[-.11 \\
.66]\end{array}$ & $\begin{array}{l}.08[-.36, \\
.47]\end{array}$ & $.16[-.33, .57]$ & $\begin{array}{l}-.05[-.50, \\
.45]\end{array}$ & $.05[-.33, .37]$ \\
\hline CLaN:L & & & & & $\begin{array}{c}.32[-.17 \\
.69]\end{array}$ & $\begin{array}{l}.13[-.23, \\
.54]\end{array}$ & $.25[-.20, .64]$ & $\begin{array}{l}.46[.08, \\
.71]^{*}\end{array}$ & $.22[-.21, .57]$ \\
\hline CLaN:DU & & & & & & $\begin{array}{l}.45[.02, \\
.75]\end{array}$ & $\begin{array}{l}-.04[-.54, \\
.45]\end{array}$ & $.17[-.32, .56]$ & $.11[-.29, .45]$ \\
\hline CLaN:A & & & & & & & $.00[-.39, .44]$ & $.02[-.39, .42]$ & $\begin{array}{l}-.22[-.58 \\
.20]\end{array}$ \\
\hline CLaN:LC & & & & & & & & $.06[-.39, .52]$ & $\begin{array}{l}-.05[-.36 \\
.31]\end{array}$ \\
\hline ISEQ & & & & & & & & & $.16[-.36, .52]$ \\
\hline
\end{tabular}

Notes. CLAN $=$ Coloured letter and number questionnaire (Rothen et al. 2013b). $\mathrm{L}=$ localization, DU $=$ deliberate use, $\mathrm{A}=$ automaticity, $\mathrm{LC}=$ longitudinal change. Consistency $=$ grapheme-colour consistency CIELUV score (Rothen et al., 2013a).

$* p<.05$

\section{Supplementary References}

Rothen, N., Seth, A. K., Witzel, C., \& Ward, J. (2013a). Diagnosing synaesthesia with online colour pickers: maximising sensitivity and specificity. Journal of Neuroscience Methods, 215(1), 156160.

Rothen, N., Tsakanikos, E., Meier, B., \& Ward, J. (2013b). Coloured Letters and Numbers (CLaN): a reliable factor-analysis based synaesthesia questionnaire. Consciousness and Cognition, 22(3), 1047-1060. 